\title{
Cobertura y solapamiento de Web of Science y Scopus en el análisis de la actividad científica española en psicología
}

\author{
Julia Osca-Lluch ${ }^{1 *}$, Sandra Miguel${ }^{2}$, Claudia González², María Peñaranda-Ortega ${ }^{3}$, Elena Quiñones-Vidal ${ }^{3}$ \\ 1 Universidad de Valencia-CSIC (España) \\ 2 Instituto de Investigaciones de Humanidades y Ciencias Sociales (dIHCS)(Argentina) \\ 3 Universidad de Murcia (España)
}

\begin{abstract}
Resumen: Este estudio tiene como primer objetivo conocer el volumen y evolución de la producción científica española en psicología a partir de las bases de datos del Web of Science (Thomson Reuters) y Scopus (Elsevier), durante el periodo 2000-2009. A partir de allí, se propone determinar cuáles son las revistas científicas más utilizadas por los psicólogos españoles para difundir sus trabajos a nivel internacional y comparar la cobertura y solapamiento de revistas y documentos que presentan ambas bases de datos. Se ofrecen datos cuantitativos, se estudia la posible correlación entre las dos bases de datos y se analiza el solapamiento de documentos y de revistas, el modelo de crecimiento que presentan y la dispersión de los trabajos, entre otros. Los resultados muestran que ambos sistemas de información son complementarios pero no excluyentes, así como la relevancia e importancia de las revistas españolas de psicología en la difusión de la investigación de esta disciplina en el contexto internacional de la ciencia.

Palabras clave: Revistas científicas; Psicología; Scopus; Web of Science; Espaก̃a.
\end{abstract}

\section{Introducción}

Las revistas académico-científicas son uno de los principales canales de comunicación y difusión de los resultados de investigación y de institucionalización social de la ciencia en la mayoría de los campos del conocimiento. Sin embargo, no todas tienen el mismo prestigio y grado de influencia en la comunidad científica. Su reconocimiento depende tanto de aspectos formales seguidos durante el proceso de edición, como del valor de su contenido científico, el que impactará con determinado grado dentro de un dominio disciplinar (Romero-Medina, 2009). Sabemos mucho acerca de las más importantes publicaciones científicas estadounidenses y anglosajonas, y quizás lo más importante para los científicos no anglosajones en la actualidad, sabemos muy bien que esta influencia se mantendrá desde el momento en que la mayoría de los que publican en ellas, pertenecen a estos ámbitos geográficos $-\mathrm{O}$ trabajan en ellos- y aunque algunas veces este interés está sobrevalorado, lo que consiguen dificultando las posibilidades de acceso al bloquear de manera continuada sus contenidos, y transformando en un estupendo negocio, la venta de los artículos sueltos que interesan a los investigadores (Aréchaga, 2007).

Los artículos de una revista profesional son el producto final de la actividad investigadora y representan la visualización de un proyecto competitivo que si quiere ser conocido internacionalmente y representar una fuente de prestigio y

* Dirección para correspondencia [Correspondence address]: Julia Osca Lluch. Instituto de Historia de la Medicina y de la Ciencia López Piñero (UV-CSIC) Palau de Cerveró, Plaza Cisneros 4, 46003, Valencia (España). E-mail: m.julia.osca@uv.es
Title: Coverage and overlap of the Web of Science and Scopus in the analysis of the Spanish scientific activity in Psychology.

Abstract: The main objective of this study is to figure out the volume and evolution of the Spanish scientific production in Psychology based in the data provided by the databases Web of Science (Thomson Reuters) and Scopus (Elsevier) during the years 2000-2009. It is proposed to determine which are the scientific journals used by most Spanish psychologists to spread their research at an international level; and to compare the coverage and overlap of the journals and documents that are present in both databases. Quantitative data is offered, as well as the possible correlation between both databases is studied and the overlap of documents and journals is analyzed, such as the increase model that is present and the dispersion of the articles, among others. The results demonstrate that both information systems are complementary but not exclusive, as well as the relevance and importance of the Spanish journals in Psychology which use the diffusion of the research in this discipline in the international science context.

Key words: Scientific journals; Psychology; Scopus; Web of Science; Spain.

de ingresos económicos para el país productor, tiene que estar hoy cercano a los presupuestos que se manejan desde las multinacionales de la edición científica. En el caso de las dos más relevantes revistas científicas (Nature y Science) sus editoras se han convertido en un formidable negocio promocionado y mantenido por sus excelentes gabinetes de prensa- que benefician exclusivamente a las compañías privadas Nature Publishing Group, y a la American Association for the Advancement of Science. Sin embargo como estas publicaciones continúan siendo el modelo que refleja la madurez científica de una disciplina, al emularlas se han aplicado los comités editoriales, los grupos investigadores y también los científicos españoles que por sus características trabajan solos, o en compañía de otros (Buela-Casal, 2009).

Así que uno de los medios para conocer el estado de la actividad investigadora y docente de cualquier institución, consiste en la evaluación de la calidad de las revistas dónde publican sus integrantes, aumentando su prestigio en función del factor de impacto que poseen éstas, independientemente del nivel de excelencia del contenido del trabajo publicado (Navarrete-Cortés, Quevedo-Blasco, ChaichioMoreno, Ríos y Buela-Casal, 2009). Los sistemas de evaluación universitarios incorporan la prospección a través de los artículos de las revistas científicas cada vez de manera más frecuente, y esto termina repercutiendo de forma directa en la evaluación de cualquier institución (Muñiz y FonsecaPedrero, 2008). Por otra parte, el número y la calidad de las publicaciones en revistas científicas es uno de los criterios para evaluar la idoneidad investigadora y docente de un profesor, un grupo de investigación, un departamento o una universidad. Se asume que los artículos publicados en revistas de prestigio son a su vez de calidad y, por tanto, influyen 
positivamente en la evaluación del currículo del autor (Carbonell y Calvó, 2009; Osca-Lluch, Civera-Mollá y Peñaranda-Ortega, 2009; Zych y Buela-Casal, 2010).

Pero el problema de la inclusión o el rechazo de determinado artículo en revistas de prestigio, y la dificultad de establecer un sistema más eficaz de selección -el de la evaluación por pares presenta muchos inconvenientes- es el principal elemento de discusión que encontramos entre partidarios y detractores del sistema vigente en la actualidad; bien sea porque los revisores mantienen su identidad oculta y no se comprometen efectivamente con los resultados del trabajo que avalan o rechazan, o porque el fraude planea en una actividad que se ha convertido en un negocio de riesgo desde que Sokal (1996) lo hizo emerger con su crítica.

Independientemente de los inconvenientes referidos, la visibilidad de las revistas científicas en los distintos repertorios o plataformas donde pueden ser vistas o buscadas, es fundamental para que sus contenidos sean conocidos y puedan ser difundidos (Abadal y Rius, 2008). Así mismo, Porque sin visibilidad se limitan las posibilidades de uso de los conocimientos generados, que es en definitiva la razón de ser de su producción (Ochoa-Henríquez, 2004). Por ello, aunque son numerosos los estudios que tratan de demostrar que la publicación de los trabajos en una revista de amplia difusión internacional o en una revista nacional no tiene por qué estar ligada necesariamente a la mayor o menor calidad de los mismos, sino que responde más bien al interés general o local de la investigación realizada, sin embargo, la visibilidad internacional de los trabajos científicos de un país constituye, en la actualidad, un indicador ampliamente utilizado como referencia del nivel científico que éste ha alcanzado y así se aplica también en España (Molina, Gómez, Cañadas, Gallardo, y Lupiáñez, 2011). El hecho de que se haya puesto en evidencia el gran avance experimentado por las revistas científicas españolas -no en castellano- incluso careciendo todavía de un plan estratégico nacional para avanzar en la profesionalización de editoras, y en la creación de consorcios de venta al exterior, no ha cristalizado en un esfuerzo consensuado con otros países castellanoparlantes, que viertan al exterior este caudal de resultados nada irrelevantes, con respecto al monto total del conocimiento, sobre todo en determinadas áreas en las que el esfuerzo financiero del Estado se ha hecho presente hasta poco antes de la crisis actual (Aréchaga, 2007; Romero Medina, 2009).

Hasta ahora, el principal y casi único indicador por el que se medía la importancia de las revistas científicas era la inclusión de las mismas en las bases de datos elaboradas por el Institute of Scientific Information (ISI) de Estados Unidos, actual Thomson Reuters (Vázquez, 2001), que se ha convertido en el cauce para lograr la consideración internacional de la investigación científica y técnica. La fuente de datos conocida como Web of Science (WoS) es una plataforma compuesta por varias bases de datos que representan en buena medida la versión electrónica de las históricas fuentes de información impresas, nacidas por separado y que siempre han tenido una doble naturaleza o finalidad: como herramienta de in- formación y como instrumento analítico subsidiario de evaluación científica (Martínez, 2008). Los productos bibliográficos elaborados desde 1960 por el Institute for Scientific Information (ISI), que actualmente forman parte del grupo empresarial Thomson Reuters, como dijimos, constituyen uno de los recursos de mayor influencia y prestigio a escala internacional.

Sin embargo, la aparición en el mercado de otra base de datos internacional y multidisciplinar de peso, Scopus, fundada por el grupo Elsevier en el año 2004 (Moya-Anegón et al., 2007) parece haber dado fin al monopolio del WoS. En la literatura más reciente han sido numerosos los estudios comparativos entre estos dos sistemas, aunque en su mayoría se refieren a aspectos generales de tipo técnico, tales como las interfaces, las facilidades para la búsqueda y otras características que presentan cada uno de ellos (Bakkalbasi, Baver, Glover y Wang, 2006; Grupo Scimago, 2006; Falagas, Pitsouni, Malietzis, y Pappas, 2008). Las comparaciones con el fin de determinar qué fuente es más apropiada para los estudios bibliométricos, no han sido concluyentes, pues las ventajas relativas de una de ellas con respecto a la otra dependen en gran medida de la disciplina y del período de análisis (López-Illescas, Moya-Anegón, y Moed., 2008, 2009; Kulkarni, Aziz, Shams, y Busse 2009; Wainer, Xavier, y Bezerra, 2009; Vieira y Gomes, 2009; Torres-Salinas, Delgado-LópezCozar, y Jiménez-Contreras, 2009; Escalona, Lagar y Pulgarín, 2010; Mingers y Lipitakis, 2010; Franceschet, 2010; Bar-Ilan, 2010; Schoegl y Gorriaiz, 2010; Mikki, 2010; Etxebarria y Gómez Uranga, 2010).

Por otra parte, y probablemente como respuesta a la nueva situación de competencia, ISI Thomson Reuters ha procedido a una importante ampliación en su cobertura internacional, incorporando un considerable número de nuevas publicaciones en la lista de fuentes que alimentan las bases del Web of Science (Rodríguez-Yunta, 2010). También se ha señalado que Scopus presenta aún importantes limitaciones, dado que no realiza un vaciado completo de todas las revistas, que los registros han sido enriquecidos con las referencias a partir de 1996 y que presenta aún algunas deficiencias en la identificación del país de procedencia de los autores. Esto último especialmente grave para las producciones de países no angloparlantes, cuyos patronímicos no son fáciles de determinar, y porque no siempre la información utiliza unos estándares procedimentales. (Jacso, 2008a, 2008b, 2008c, 2010).

La dificultad para determinar las características de la producción disciplinar total de un país debido a la falta de instrumentos de registro, hace que los estudios de actividad científica se basen en estas fuentes por considerar que, pese a sus limitaciones, contienen en su seno la referencia a los principales canales de confianza de la comunicación científica internacional (Carbonell y Calvó, 2009; Olivas-Ávila, Musi-Lechuga, Guillén-Riquelme y Castro, 2012). Y, aunque insistimos en el interés de crear una plataforma que reúna la producción científica en otros idiomas, siquiera bilingüe, por 
el ahorro sustancial de esfuerzos y sinergias, nos remitiremos a las técnicas al uso.

Así en el presente trabajo, nos proponemos conocer cuáles son las revistas de psicología con visibilidad internacional, en las que publican los investigadores de esta disciplina que trabajan en alguna institución española, cuáles son las más utilizadas y qué grado de solapamiento existe entre ambas bases de datos (WoS y Scopus) en el área temática propuesta, tanto en lo que se refiere a la cobertura de títulos de revistas como de documentos, y cuáles son los países de edición de las revistas en las que los investigadores españoles del área de psicología publican con más frecuencia.

\section{Material y métodos}

El estudio se ha realizado utilizando como fuentes de información las bases de datos Science Citation Index (SCI), Social Science Citation Index (SSCI) y Journal Citation Report (JCR), de la ISI Web of Science (WoS) y la base de datos Scopus de Elsevier.

Equiparar y verificar las sensibilidades de las diferentes bases de datos es una tarea que representa algunas dificultades, como consecuencia de que cada base de datos tiene su propio sistema de indagación. Las ecuaciones de búsqueda ejecutadas, con el fin de poder recuperar los trabajos de psicología realizados en alguna institución española, tanto en WoS como en Scopus han sido las siguientes:

En las bases de datos $W_{O} S$, se ha realizado una ecuación de búsqueda relacionando todas las revistas recogidas en el JCR (del SCI y SSCI) incluidas en las diferentes categorías de psicología, e interrogando a las bases de datos SCI y SSCI combinándolo con el término Spain en el campo "Address" y el período de años 2000-2009 en el campo "Year Published". La delimitación temática se hizo atendiendo a la clasificación de revistas en disciplinas elaborada por Thomson Scientific, de forma que se consideraron documentos de psicología aquellos publicados en alguna de las revistas incluidas en las disciplinas "Psychology", "Psychology Applied", "Psychology Biological", "Psychology Clinical", Psychology Developmental", "Psychology Educational", "Psychology Experimental", "Psychology Mathematical", "Psychology Multidisciplinary", "Psychology Social” y Psychology Psychoanalysis".

Para la búsqueda en Scopus, se seleccionaron todos los trabajos incluidos en el campo "Subjarea" Psychology (Psicología), combinándolo con el término Spain en el campo "Affilcountry" y limitándolo a los años 2000-2009 en el campo "Published".

El grado de solapamiento entre las dos bases de datos se ha calculado a partir de la identificación de los documentos y los títulos de las revistas comunes a ambas fuentes.

\section{Resultados}

\section{Cobertura y solapamiento de documentos entre Scopus y WoS}

La consulta a las diferentes bases de datos analizadas dio como resultado un total de 14216 documentos. Su distribución por base de datos arrojó un número de documentos muy similar; en WoS (SCI y SSCI) se recuperaron 7260 documentos y en Scopus, 6956.

En la Figura 1 se muestra la distribución por años del número de trabajos recogidos en cada una de las bases de datos. En ambas se observa un crecimiento constante, especialmente en Scopus. Llama la atención el incremento de trabajos españoles indizados en el año 2008 por la base de datos WoS; sin embargo, se trata de un aumento que podríamos clasificar como anecdótico, ya que es como consecuencia de la indización de un número monográfico publicado en ese año.

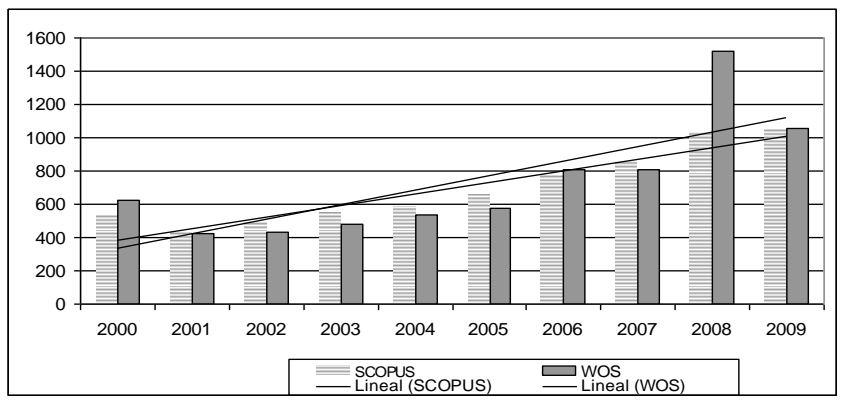

Figura 1. Evolución del número de trabajos por años.

Las dos bases de datos analizadas proporcionan un total de 9794 documentos diferentes. En total, 5431 documentos $(55.45 \%)$ son documentos únicos, recogidos en una sola de las bases de datos y $4363(44.55 \%)$ están solapados entre las dos.

Del total de documentos únicos, es decir, que son recogidos solamente por una de las dos bases de datos consultadas, $2.890(29.51 \%$ ) han sido recuperados de la WoS y 2541 (25.94\%) de Scopus. En la figura 2 se presenta la distribución por años del número de trabajos y solapamientos entre ellas.

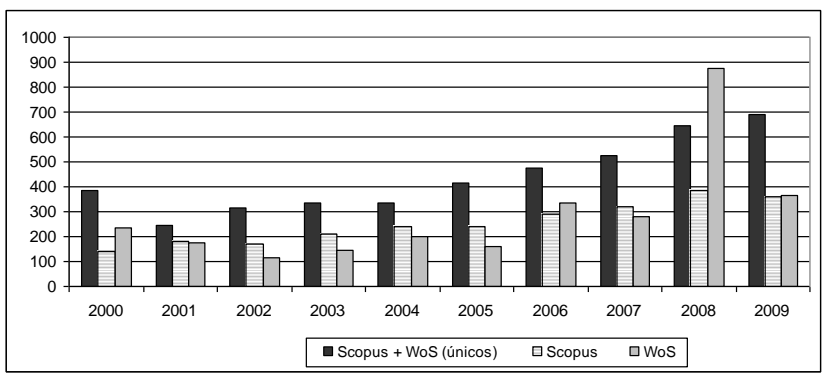

Figura 2. Número de trabajos por años y solapamiento (2000-2009). 


\section{Cobertura y solapamiento de revistas entre Scopus y WoS}

El número total de revistas en las que han publicado artículos los investigadores del área de psicología que trabajan en alguna institución española durante el período estudiado, y que han sido recogidos por alguna de las bases de datos consultadas (SCI, SSCI y Scopus) es de 607.

El 57\% de las revistas mostradas (351 títulos) están solapadas entre las dos bases de datos consultadas, mientras que casi el 43\% (256 títulos) son recogidas solamente por una de las dos bases de datos (ver Figura 3).

La base de datos que abarca un mayor número de revistas de psicología elegidas por los investigadores que trabajan en alguna institución española durante el período estudiado es Scopus (555). De éstas 204 son revistas exclusivas de esta fuente. En las bases de datos del WoS (SCI y SSCI), el número total de revistas asciende a 403, de las que 351 están solapadas con $S$ copus y 52 circulan solamente en WoS.

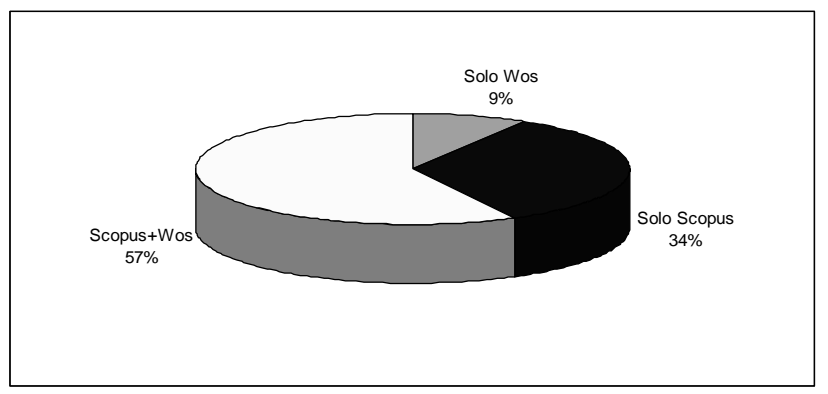

Figura 3. Distribución del número de revistas por bases de datos (20002009).

Cobertura de las bases de datos según país de edición de las revistas

Cuando se analiza el país de procedencia de las revistas incluidas en las dos bases de datos, se observan algunas diferencias significativas. En la Tabla 1 se muestra la distribución del número de revistas donde han publicado los investigadores españoles, según su país de edición. El número total de países es de 25, destacando Estados Unidos, como el país editor de un mayor número de revistas en las dos bases de datos consultadas (312), con un porcentaje de más del $50 \%$ de las revistas, seguido de Reino Unido (139) y Holanda (52).

Basta la comparación entre las diferentes columnas para darse cuenta que la distribución por países de las revistas de psicología utilizadas por los investigadores españoles no es uniforme en las dos bases de datos consultadas. Quizá el hecho más destacado es que la base de datos Scopus recoge revistas procedentes de un mayor número de países que $W o S$ y que aunque el país con un mayor número de revistas de psicología indizadas es Estados Unidos, su peso con respecto al total es inferior que en la $W o S(49.73 \%$ frente al
$59.06 \%$ ). En la base de datos $W_{0} S$, Estados Unidos y Reino Unido son los países de edición del mayor número de revistas de psicología donde han publicado los investigadores españoles durante el período analizado (83.87\%), mientras que en Scopus el porcentaje de estos dos países suponen el $73.15 \%$ del total. En lo que respecta a España, vemos que tanto en Scopus (18 revistas) como en $W$ os ( 2 revistas) ocupa el cuarto puesto junto con Alemania. Le sigue muy de cerca Francia, con 17 revistas en total.

Tabla 1. País de edición y solapamiento entre las revistas.

\begin{tabular}{|c|c|c|c|c|}
\hline Países & $\begin{array}{l}\mathrm{N}^{o} \text { revistas } \\
\text { solo-Scopus }\end{array}$ & $\begin{array}{c}\mathrm{N}^{o} \text { revistas } \\
\text { solo- } W_{0} S\end{array}$ & $\begin{array}{c}\mathrm{N}^{\circ} \text { revistas } \\
\text { solapadas } \\
(\text { Scopus y } W o s)\end{array}$ & $\begin{array}{l}\text { Total- } \\
\text { revistas } \\
\text { por-país }\end{array}$ \\
\hline Alemania & 7 & 0 & 11 & 18 \\
\hline Argentina & 0 & 0 & 1 & 1 \\
\hline Australia & 0 & 1 & 1 & 2 \\
\hline Bélgica & 0 & 0 & 1 & 1 \\
\hline Brasil & 7 & 0 & 1 & 8 \\
\hline Canadá & 2 & 0 & 2 & 4 \\
\hline Chile & 2 & 0 & 1 & 3 \\
\hline Colombia & 3 & 0 & 1 & 4 \\
\hline Corea & 1 & 0 & 0 & 1 \\
\hline Eslovaquia & 0 & 0 & 1 & 1 \\
\hline España & 9 & 2 & 9 & 20 \\
\hline Estados Unidos & 74 & 36 & 202 & 312 \\
\hline Francia & 14 & 1 & 2 & 17 \\
\hline Grecia & 2 & 0 & 0 & 2 \\
\hline Holanda & 31 & 2 & 19 & 52 \\
\hline Italia & 2 & 0 & 1 & 3 \\
\hline Japón & 0 & 0 & 1 & 1 \\
\hline México & 2 & 0 & 1 & 3 \\
\hline Noruega & 1 & 0 & 0 & 1 \\
\hline Nueva Zelanda & 1 & 0 & 1 & 2 \\
\hline Polonia & 1 & 0 & 0 & 1 \\
\hline Portugal & 0 & 0 & 1 & 1 \\
\hline Reino Unido & 39 & 9 & 91 & 139 \\
\hline Suecia & 1 & 0 & 0 & 1 \\
\hline Suiza & 5 & 1 & 3 & 9 \\
\hline Total revistas & 204 & 50 & 351 & 607 \\
\hline
\end{tabular}

\section{Dispersión de los trabajos}

Los trabajos científicos de una disciplina cualquiera no se distribuyen equilibradamente entre un número determinado de revistas, sino que es posible detectar en todas las materias una concentración mayor de artículos en un conjunto limitado de revistas. En este trabajo, se observa que la revista más utilizada por los investigadores españoles durante todo el periodo estudiado es Psicothema, siendo la que lidera el ranking por registrar un mayor número de trabajos recogidos en ambas fuentes.

En la Tabla 2 se muestra la relación de las revistas indizadas por las bases de datos Scopus y/o WoS donde han publicado un mayor número de trabajos los investigadores españoles durante el período 2000-2009. 
Tabla 2. Relación de revistas indizadas por Scopus y Wos donde han publicado un mayor número de trabajos los investigadores españoles (2000-2009).

\begin{tabular}{|c|c|c|c|c|c|c|c|}
\hline 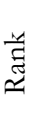 & SCOPUS & 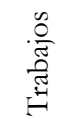 & $\%$ & & WOS & 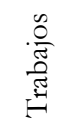 & $\%$ \\
\hline 1 & Psicothema & 1138 & 16.49 & 1 & Psicothema & 1431 & 19.34 \\
\hline 2 & Spanish Journal of Psychology & 202 & 2.93 & 2 & International Journal of Psyhology & 690 & 9.32 \\
\hline 3 & Behavioral Psychology-Psicología conductual & 197 & 2.85 & 3 & Psycho-Oncology & 255 & 3.45 \\
\hline 4 & $\begin{array}{l}\text { International Journal of Clinical and Health } \\
\text { Psychology }\end{array}$ & 142 & 2.06 & 4 & Spanish Journal of Psychology & 207 & 2.80 \\
\hline 5 & $\begin{array}{l}\text { International Journal of Psychology and Psy- } \\
\text { chological Therapy }\end{array}$ & 127 & 1.84 & 5 & Psychophysiology & 168 & 2.27 \\
\hline 5 & Personality and Individual Differences & 127 & 1.84 & 6 & Journal of Psychophysiology & 163 & 2.20 \\
\hline 6 & Archivos de Psiquiatria & 117 & 1.70 & 7 & $\begin{array}{l}\text { International Journal of Clinical and Health Psy- } \\
\text { chology }\end{array}$ & 145 & 1.96 \\
\hline 7 & Psychological Reports & 94 & 1.36 & 8 & Personality and Individual Differences & 126 & 1.70 \\
\hline 8 & Journal of Affective Disorders & 90 & 1.30 & 9 & Infancia y Aprendizaje & 118 & 1.59 \\
\hline 8 & Schizophrenia Research & 90 & 1.30 & 10 & Perception & 117 & 1.58 \\
\hline 9 & Revista Latinoamericana de Psicología & 88 & 1.28 & 11 & Revista de Psicología del Deporte & 107 & 1.45 \\
\hline 10 & Journal of Clinical Psychiatry & 68 & .99 & 12 & Revista Latinoamericana de Psicología & 105 & 1.42 \\
\hline & $\begin{array}{l}\text { Journal of Neurology, Neurosurgery and Psy- } \\
\text { chiatry }\end{array}$ & 67 & .97 & 13 & Revista Mexicana de Psicología & 102 & 1.38 \\
\hline 12 & Papeles del Psicólogo & 65 & .94 & 14 & Psychological Reports & 101 & 1.36 \\
\hline 12 & Perceptual and Motor Skills & 65 & .94 & 15 & Journal of Clinical Psychiatry & 96 & 1.30 \\
\hline 13 & Revista Mexicana de Psicología & 60 & .87 & 16 & Behavioral Psychology-Psicología conductual & 94 & 1.27 \\
\hline 14 & European Journal of Psychological Assessment & 57 & .83 & 17 & Anales de Psicología & 89 & 1.20 \\
\hline 15 & Psicológica & 54 & .78 & 18 & International Journal of Psychophysiology & 85 & 1.15 \\
\hline 16 & Journal of Alzheimer's Disease & 51 & .74 & 19 & Cyberpsychology \& Behavior & 80 & 1.08 \\
\hline 17 & Neurobiology of Aging & 47 & .68 & 19 & Physiology \& Behavior & 80 & 1.08 \\
\hline 18 & Psicooncología & 46 & .67 & 20 & Aggressive Behavior & 75 & 1.01 \\
\hline 19 & Psychiatry Research & 45 & .65 & 21 & Perceptual and Motor Skills & 71 & .96 \\
\hline 20 & Behavior Research Methods & 41 & .59 & 22 & Revista de Psicodidáctica & 66 & .89 \\
\hline 20 & Pain & 41 & .59 & 23 & European Journal of Psychological Assessment & 61 & .82 \\
\hline & Neuropsychologia & 39 & .57 & 24 & Psicológica & 61 & .82 \\
\hline & $\begin{array}{l}\text { Journal of Neurology Neurosurgery and Psy- } \\
\text { chiatry }\end{array}$ & 36 & .52 & 25 & Revista de Psicología Social & 50 & .68 \\
\hline 22 & Quarterly Journal of Experimental Psychology & 36 & .52 & 26 & Psychology \& Health & 46 & .62 \\
\hline 23 & Journal of Pragmatics & 34 & .49 & 27 & Behavior Research Methods & 43 & .58 \\
\hline & European Neuropsychopharmacology & 33 & .48 & 28 & International Journal of Eating Disorders & 41 & .55 \\
\hline 24 & Journal of Human Movement Studies & 33 & .48 & 29 & Estudios de Psicología & 40 & .54 \\
\hline 24 & Revista de Psicologia del Deporte & 33 & .48 & 30 & Neuropsychologia & 39 & .53 \\
\hline 25 & Aggressive Behavior & 32 & .46 & 31 & International Psychogeriatrics & 38 & .51 \\
\hline 25 & Bipolar Disorders & 32 & .46 & 31 & International Journal of Psychoanalysis & 37 & .5 \\
\hline 25 & Dementia and Geriatric Cognitive Disorders & 32 & .46 & 31 & Quarterly Journal of Experimental Psychology & 36 & .49 \\
\hline
\end{tabular}

Hay que destacar que en algunas revistas que están incluidas en las dos bases de datos (Scopus y WoS) el número de documentos recogidos en cada una de ellas varía considerablemente. Es decir, algunas revistas están recogidas en las dos bases de datos, pero sin embargo, no ofrecen información sobre el mismo número de trabajos. Tres podrían ser los motivos que explican estas diferencias; En primer lugar, las distintas políticas de indización de las bases de datos, que difieren en su cobertura de los diferentes tipos documentales, y que deben tenerse en cuenta en los estudios de solapamiento. En segundo lugar, se observa que existen cambios en la cobertura de las bases de datos y la permanencia de algunas revistas en ellas no es continua, sino que puede variar a lo largo del tiempo. En tercer lugar, las limitaciones que aún presenta Scopus en la identificación del país de procedencia de los autores, que podría en este caso dificultar la búsqueda de todos los investigadores que trabajan en instituciones españolas y que han publicado en revistas incluidas por esa fuente.

Con el fin de ilustrar con mayor detalle los cambios de cobertura y permanencia de las revistas en las bases de datos, en la Tabla 3 se ofrece la relación de revistas españolas de psicología que han sido indizadas en $S c o p u s$ y $W o S$, junto con sus años de permanencia. Tal como se puede observar, solamente hay una, Psicothema, que es indizada por Scopus y WoS durante todo el período 2000-2009 y tres revistas (International Journal of Clinical and Health Psychology, Psicologica y Spanish Journal of Psychology) que son recogidas ininterrumpida- 
mente por las dos bases de datos desde el año 2005. Se observa un incremento de la presencia del número de revistas españolas indizadas en estas dos bases de datos, sobre todo en los últimos años del período analizado.

Tabla 3. Evolución de las revistas españolas de psicología indizadas por Scopus y WoS (2000-2009).

\begin{tabular}{|c|c|c|c|c|c|c|c|c|c|c|c|c|c|c|c|c|c|c|c|c|}
\hline & 20 & & & & & & & & & & & & & & & & & & & \\
\hline Títulos de las revistas & $\mathrm{S}$ & W & $\mathrm{S}$ & $\mathrm{W}$ & $\mathrm{S}$ & $\mathrm{W}$ & $\mathrm{S}$ & $\mathrm{W}$ & $\mathrm{S}$ & $\mathrm{W}$ & $\mathrm{S}$ & W & $\mathrm{S}$ & $\mathrm{W}$ & $\mathrm{S}$ & $\mathrm{W}$ & $\mathrm{S}$ & W & $\mathrm{S}$ & W \\
\hline Anales de Psicología & & & & & & & & & & & & & & & & $\mathrm{x}$ & & $\mathrm{x}$ & $\mathrm{x}$ & $\mathrm{x}$ \\
\hline Ansiedad y Estrés & & & & & & & & & & & & & & & & & & & $\mathrm{x}$ & \\
\hline Anuario de Psicología & & & & & & & & & & & & & & & & & $\mathrm{x}$ & & $\mathrm{x}$ & \\
\hline Archivos de Psiquiatría & $\mathrm{x}$ & & $\mathrm{x}$ & & $\mathrm{x}$ & & $\mathrm{x}$ & & $\mathrm{x}$ & & $\mathrm{x}$ & & $\mathrm{x}$ & & $\mathrm{x}$ & & & & & \\
\hline $\begin{array}{l}\text { Behavioral Psychology/ Psicología Con- } \\
\text { ductual }\end{array}$ & $\mathrm{x}$ & $\mathrm{x}$ & $\mathrm{x}$ & & $\mathrm{x}$ & & $\mathrm{x}$ & & $\mathrm{x}$ & & $\mathrm{x}$ & & $\mathrm{x}$ & & $\mathrm{x}$ & $\mathrm{x}$ & $\mathrm{x}$ & $\mathrm{x}$ & $\mathrm{x}$ & $\mathrm{x}$ \\
\hline $\begin{array}{l}\text { Electronic Journal of Research in Educa- } \\
\text { tional Psychology }\end{array}$ & & & & & & & & & & & & & & & & & & & $\mathrm{x}$ & \\
\hline Estudios de Psicología & & & & & & & & & & & & & & & & & & $\mathrm{x}$ & & $\mathrm{x}$ \\
\hline Goze & & & & & & & & & & & $\mathrm{x}$ & & & & & & & & & \\
\hline Infancia y Aprendizaje & & & & & & & & & & & & & $\mathrm{x}$ & $\mathrm{x}$ & $\mathrm{x}$ & $\mathrm{x}$ & $\mathrm{x}$ & $\mathrm{x}$ & $\mathrm{x}$ & $\mathrm{x}$ \\
\hline $\begin{array}{l}\text { International Journal of Clinical and } \\
\text { Health Psychology }\end{array}$ & & & & & & & & & & & $\mathrm{x}$ & $\mathrm{x}$ & $\mathrm{x}$ & $\mathrm{x}$ & $\mathrm{x}$ & $\mathrm{x}$ & $\mathrm{x}$ & $\mathrm{x}$ & $\mathrm{x}$ & $\mathrm{x}$ \\
\hline $\begin{array}{l}\text { International Journal of Psychology and } \\
\text { Psychological Therapy }\end{array}$ & & & $\mathrm{x}$ & & $\mathrm{x}$ & & $\mathrm{x}$ & & $\mathrm{x}$ & & $\mathrm{x}$ & & $\mathrm{x}$ & & $\mathrm{x}$ & & $\mathrm{x}$ & & $\mathrm{x}$ & \\
\hline Papeles del Psicólogo & & & & & & & & & & & & & & & $\mathrm{x}$ & & $\mathrm{x}$ & & $\mathrm{x}$ & \\
\hline Psicológica & & & & & & & & & $\mathrm{x}$ & & $\mathrm{x}$ & $\mathrm{x}$ & $\mathrm{x}$ & $\mathrm{x}$ & $\mathrm{x}$ & $\mathrm{x}$ & $\mathrm{x}$ & $\mathrm{x}$ & $\mathrm{x}$ & $\mathrm{x}$ \\
\hline Psicooncologia & & & & & & & & & & & & & & & & & $\mathrm{x}$ & & $\mathrm{x}$ & \\
\hline Psicothema & $\mathrm{x}$ & $\mathrm{x}$ & $\mathrm{x}$ & $\mathrm{x}$ & $\mathrm{x}$ & $\mathrm{x}$ & $\mathrm{x}$ & $\mathrm{x}$ & $\mathrm{x}$ & $\mathrm{x}$ & $\mathrm{x}$ & $\mathrm{x}$ & $\mathrm{x}$ & $\mathrm{x}$ & $\mathrm{x}$ & $\mathrm{x}$ & $\mathrm{x}$ & $\mathrm{x}$ & $\mathrm{x}$ & $\mathrm{x}$ \\
\hline Revista de Psicodidáctica & & & & & & & & & & & & & & & & $\mathrm{x}$ & $\mathrm{x}$ & $\mathrm{x}$ & $\mathrm{x}$ & $\mathrm{x}$ \\
\hline Revista de Psicología del Deporte & & & & & & & & & & & & & & & & $\mathrm{x}$ & $\mathrm{x}$ & & $\mathrm{x}$ & $\mathrm{x}$ \\
\hline Revista de Psicología Social & & & & & & & & & & & & & & & & & & $\mathrm{x}$ & & $\mathrm{x}$ \\
\hline Salud Mental & & & & & & & & & & & & & & & & & $\mathrm{x}$ & & $\mathrm{x}$ & \\
\hline Spanish Journal of Psychology & $\mathrm{x}$ & & $\mathrm{x}$ & & $\mathrm{x}$ & & $\mathrm{x}$ & & $\mathrm{x}$ & & $\mathrm{x}$ & $\mathrm{x}$ & $\mathrm{x}$ & $\mathrm{x}$ & $\mathrm{x}$ & $\mathrm{x}$ & $\mathrm{x}$ & $\mathrm{x}$ & $\mathrm{x}$ & $\mathrm{x}$ \\
\hline
\end{tabular}

\section{Discusión}

En la actualidad, las dos bases de datos más utilizadas para los estudios bibliométricos que buscan conocer la situación de la investigación en una disciplina son la Web of Science (WoS), de la empresa Thomson Reuters, y Scopus, del grupo Elsevier. Ambas son de alcance internacional, multidisciplinar y con una amplia cobertura de revistas.

Los resultados obtenidos en el análisis comparativo de las dos bases de datos Scopus y WoS, han mostrado que los dos sistemas son muy similares, siendo complementarios de cara a su cobertura de revistas y de documentos publicados por parte de los investigadores españoles de psicología. Esta similitud se ha podido establecer comparando la producción científica anual en ambos sistemas, así como el número de revistas durante el período 2000-2009. Existe una tendencia claramente ascendente de la producción científica en los dos sistemas y un alto porcentaje de solapamiento en ambas fuentes, de manera similar a lo que ocurre en otras disciplinas científicas (Escalona et al., 2010).

Sin embargo, el estudio muestra que a pesar de las similitudes, existen diferencias en la cobertura de ambas bases de datos, encontrando que en algunas revistas que están recogidas por las dos fuentes (Scopus y WoS) el número de documentos recogidos en cada una de ellas puede variar, en parte, como consecuencia de las distintas políticas de indización de las bases de datos, cambios en la cobertura (altas y bajas de revistas) y limitaciones producto de la calidad de los datos que pueden afectar la representación de la producción.

Esta información se presupone útil para comprender las diferencias en los resultados de estudios bibliométricos que utilicen WoS o Scopus como fuente de datos y complementar los trabajos existentes sobre la cobertura de revistas y de la producción recogida en estas fuentes.

También constatamos el aumento ciertamente llamativo del número de publicaciones españolas en los últimos diez años, coincidiendo con el aumento de la financiación para el avance de la investigación realizados en nuestro país en la última década: 9794 documentos hablan positivamente del convencimiento de los psicólogos de que hay que ampliar los dominios y hacerse visibles de manera intensa y progresiva, aunque sólo el $44.55 \%$ de ellos estén solapados entre las dos bases de datos, lo que quiere decir que hay que esforzarse más en que la presencia de los mismos se multiplique por dos.

Como hemos puesto de manifiesto, la revista Psicothema es la más representada en ambas bases, lo que no es extraño desde el momento en que fue la primera en someterse tanto a las directrices formales de las editoriales como a la cuestión de los contenidos y distribución del conocimiento, admitiendo trabajos que estén referidos a la aplicación de una técnica, que enfatizaran la utilidad de los resultados, y la contundencia de los argumentos, así como asumir la tarea editorial de determinar la importancia de los resultados y adelantar una publicación. El éxito inicial de Psicothema propició la 
adopción de las condiciones de publicación de otras revistas (International Journal of Clinical and Health Psychology, Psicológica y Spanish Journal of Psychology) que son recogidas ininterrumpi- damente por las dos bases de datos desde el año 2005, y que han sido seguidas por otras más, las cuales han entrado con fuerza en el ranking de revistas a considerar.

\section{Referencias}

Abadal, E. y Rius Alcaraz, L. (2008). Revistas científicas de las universidades españolas: acciones básicas para aumentar su difusión e impacto. Revista Española de Documentación Cientifica, 31(2), 242-262.

Aréchaga, J. (2007, 26 diciembre). Revistas científicas en España: Entre el aldeanismo y el desdén. El País, pp. A40.

Bakkalbasi, N., Baver, K., Glover, J. y Wang., L. (2006). Three options for citation tracking: Google Schoolar, Scopus and Web of Science. BMC. Biomedical Digital Libraries, 3(7), 1-8.

Bar-Ilan, J. (2010). Citations to the "Introduction to Informetrics" indexed by WOS, Scopus and Google Scholar. Scientometrics, 82(3), 495-506.

Carbonell, X. y Calvó, N. (2009). Las revistas españolas de psicología: cómo elegir la revista donde publicar. Anales de Psicología, 25(2), 209-216.

Escalona Fernández, M. I., Lagar Barbosa, P. y Pulgarín Guerrero, A. (2010). Web of Science vs. Scopus: un estudio cuantitativo en ingeniería química. Anales de Documentación, 13, 159-175.

Etxebarria, G. y Goméz-Uranga, M. (2010). Use of Scopus and Google Scholar to measure social sciences production in four major Spanish universities. Scientometrics, 82(2), 333-349.

Falagas, M. E., Pitsouni, E. I., Malietzis, G. A., y Pappas, G. (2008). Comparison of Pubmed, Scopus, Web of Science, and Google Schoolar: strengths and weaknesses. The Faseb Journal, 22, 338-342.

Franceschet, M. (2010). A comparison of bibliometric indicators for computer science scholars and journals on Web of Science and Gogle Scholar. Scientometrics, 81(1), 243-258.

Grupo Scimago (2006). Análisis de la cobertura de la base de datos Scopus. El Profesional de la Información, 15(2), 144-145.

Jacso, P. (2008a). Testing the calculation of a realistic h-index in Google Scholar, Scopus, and Web of Science for F. W. Lancaster. Library Trends, 56(4), 784-815.

Jacso, P. (2008b). The pros and cons of computing the h-index using Scopus. Online Information Review, 32(4), 524-535.

Jacso, P. (2008c). The plausibility of computing the h-index of scholarly productivity and impact using reference-enhanced databases. Online Information Review, 32(2), 266-283.

Jacso, P. (2010). Comparison of journal impact rankings in the SCImago Journal \& Country Rank and the Journal Citation Reports databases. Online Information Review, 34 (4), 642-657.

Kulkarni, A.V., Aziz, B., Shams, I. y Busse, J. W. (2009). Comparisons of citations in Web of Science, Scopus, and Google Scholar for articles published in General Medical Journals. The Journal of the American Medical Association, 302(10), 1092-1096.

López-Illescas, C.; Moya-Anegón, F. y Moed, H. F. (2008). Coverage and citation impact of oncological journals in the Web of Science and Scopus. Journal of Informetrics, 3, 304-316.

López-Illescas, C.; Moya-Anegón, F. y Moed, H. F. (2009). Comparing bibliometric country-by-country rankings derived from the Web of Science and Scopus: the effect of poorly cited journal in oncology. Journal of Information Science, 35, 244-256.

Martínez, L. J. (2008). La nueva versión de ISI Web of Knowledge: calidad y complejidad. El Profesional de la Información, 17(3), 331-339.

Mikki, S. (2010). Comparing Google Scholar and ISI Web of Science for Earth Sciences. Scientometrics, 82(2), 321-331.

Mingers, J. y Lipitakis, L. E. A. (2010). Counting the citations: a comparison of Web of Science and Google Scholar in the field of business and management. Scientometrics, 85(2), 613-625.
Molina, M., Gómez, P., Cañadas, M. C., Gallardo, J. y Lupiáñez, J. L. (2011). Calidad y visibilidad de las revistas científicas: el caso de PNA. Revista Española de Documentación Cientifica, 34(2), 266-275.

Moya-Anegón, F., Chinchilla-Rodríguez, Z., Vargas-Quesada, B., CorreaÁlvarez, E., Muñoz-Fernández, F. J., González-Molina, A. y HerreroSolana, A. (2007). Coverage analysis of Scopus: A journal metric approach. Scientometrics, 73(1), 53-78.

Muñiz, J. y Fonseca-Pedrero, E. (2008). Construcción de instrumentos de medida para la evaluación universitaria. Revista de Investigación en Educación, 5, 13-25.

Navarrete-Cortes, J., Quevedo-Blasco, R., Chaichio-Moreno, J. A., Ríos, C. y Buela-Casal, G. (2009). Análisis cuantitativo por países de la productividad en psicología de las revistas en la Web of Science. Revista Mexicana de Psicología, 26(2), 131-143.

Ochoa-Henríquez, H. (2004). Visibilidad: el reto de las revistas científicas latinoamericanas. Opción, 43, 162-168.

Olivas-Ávila, J. A., Musi-Lechuga, B., Guillén-Riquelme, A. y Castro, A. (2012). Diferencias en la producción investigadora en tesis y artículos de los profesores funcionarios de psicología en España en función del sexo. Anales de Psicología, 28, 597-603.

Osca-Lluch, J., Civera-Mollá, C. y Peñaranda-Ortega, M. (2009). Consecuencias de los errores en las referencias bibliográficas. El caso de la revista Psicothema. Psicothema, 21, 300-303.

Rodríguez-Yunta, L. (2010). Las revistas iberoamericanas en Web of Science y Scopus: visibilidad internacional e indicadores de calidad. En J. Ríos Ortega (Coord.), VII Seminario Hispano-Mexicano de Investigación en Bibliotecología y Documentación, Ciudad de México, 7-9 de abril de 2010. Memoria del $7^{\circ}$ Seminario Hispano-Mexicano de Investigación en Bibliotecología y Documentación (347-363) México: UNAM, Centro Universitario de Investigaciones Bibliotecológicas.

Romero Medina, A. (2009). Evolución de la calidad y difusión de una revista científica española de Psicología: Anales de Psicología, 25 volúmenes anuales publicados (1984-2009). Anales de Psicología, 25(2), 181-198.

Schoegl, C. y Gorriaiz, J. (2010). Comparison of citation and usage indicators: the case of oncology journals. Scientometrics, 82(3), 567-580.

Sokal, A. D. (1996). Transgresing the boundaries: Towards a transformative hermeneutics of quantum gravity. Social Text, 46-47, 217-252.

Torres-Salinas, D., Delgado-López-Cózar, E., y Jiménez-Contreras, E. (2009). Ranking of departments and researchers within a university using two different databases: Web of Science versus Scopus. Scientometrics, $80(3), 761-774$

Vázquez Valero, M. (2001). Las revistas nacionales y los estudios de usuarios.Forinf@, 14, 4-5.

Vieira, E. S. y Gomes, J. A. N. F. (2009). A comparison of Scopus and Web of Science for a typical university. Scientometrics, 81(2), 587-600.

Wainer, J., Xavier, E. C. y Bezerra, F. (2009). Scientific production in Computer Science: a comparative study of Brazil and other countries. Scientometrics, 81(2), 535-547.

Zych, I. y Buela-Casal, G. (2010). Internacionalidad de las revistas de psicología multidisciplinar editadas en Iberoamérica e incluidas en la Web of Science. Universitas Psychologica, 9(1), 27-34.

(Artículo recibido: 26-6-2012, revisado: 8-2-2013, aceptado: 22-2-2013) 